\title{
Research Article \\ Detection of Short-Step Pulses Using Practical Test-Functions and Resonance Aspects
}

\author{
Alexandru Toma and Cristian Morarescu \\ Corner Soft Technologies, 23 George Macarovici St., Bucharest 6, 060142, Romania \\ Correspondence should be addressed to Cristian Morarescu,c1mora2@yahoo.com
}

Received 16 May 2008; Accepted 3 June 2008

Recommended by Ming Li

An important aspect in modeling dynamic phenomena consists in measuring with higher accuracy some physical quantities corresponding to the dynamic system. Yet for measurements performed on limited time interval at high working frequency, certain intelligent methods should be added. The high working frequency requires that the measurement and data processing time interval should be greater than the time interval when the step input is received, so as to allow an accurate measurement. This paper will show that an intelligent processing method based on oscillating second-order systems working on limited time interval can differentiate between large step inputs (which are active on the whole limited time interval) and short step inputs (which are active on a time interval shorter than the limited working period). Some resonance aspects (appearing when the input frequency is close to the working frequency of the oscillating second-order system) will be also presented.

Copyright (C) 2008 A. Toma and C. Morarescu. This is an open access article distributed under the Creative Commons Attribution License, which permits unrestricted use, distribution, and reproduction in any medium, provided the original work is properly cited.

\section{Introduction}

Filtering and sampling devices usually consist of asymptotically stable systems, sometimes an integration of the output over a certain time interval being added. Yet such structures are very sensitive at random variations of the integration period, being recommended for the signal which is integrated to be approximately equal to zero at the end of the integration period. For this reason, oscillating systems for filtering the received signal should be used, so as the filtered signal and its slope to be approximately zero at the end of a certain time interval (at the end of an oscillation). For avoiding instability of such oscillating systems on extended time intervals, certain electronic devices (gates) controlled by computer commands should be added, so as to restore the initial null conditions for the oscillating system before a new working cycle to start [1].

The filtering performances of asymptotically stable systems are determined by their transfer function. A filtering and sampling device consisting of low-pass filters of first or 
second order having the transfer function

$$
H(s)=\frac{1}{T_{0} s+1}
$$

(for a first-order system) and

$$
H(s)=\frac{1}{T_{0}^{2} s^{2}+2 b T_{0} s+1}
$$

(for a second-order system) attenuates an alternating signal of angular frequency $\omega \gg \omega_{0}=$ $1 / T_{0}$ about $\omega / \omega_{0}$ times (for a first-order system) or about $\left(\omega / \omega_{0}\right)^{2}$ times (for a second-order system). The response time of such systems at a continuous useful signal is about $4-6 T_{0}\left(5 T_{0}\right.$ for the first-order system and $4 T_{0} / b$ for the second-order system). If the signal given by the first- or second-order system is integrated over such a period, a supplementary attenuation for the alternating signal of about $4-6 \omega / \omega_{0}$ can be obtained.

But such structures are very sensitive at the random variations of the integration period (for unity-step input, the signal which is integrated is equal to unity at the sampling moment of time), and the use of oscillators with a very high accuracy cannot solve the problem due to switching phenomena appearing at the end of the integration period (when an electric current charging a capacitor is interrupted).

These random variations cannot be avoided if we use asymptotically stable filters. By the other hand, an improvement in an electrical scheme used for integrators in analog signal processing (see $[2,3]$ ) cannot lead to a significant increasing in accuracy, as long as such electronic devices perform the same task (the system has the same transfer function). There are also known techniques for reducing the switching noise in digital systems, but such procedures can be applied only after the analog signal is filtered and sampled, so as to be prepared for further processing. So we must give attention to some other kind of transfer functions and to analyze their properties in case of filtering and sampling procedures, similar to wavelets analysis presented in $[4,5]$.

Mathematically, an ideal solution consists in using an extended Dirac function for multiplying the received signal before the integration (see [1]), but is very hard to generate such extended Dirac functions (a kind of acausal pulses) using nonlinear differential equations for (i) symmetrical pulses (see [6]) or (ii) asymmetrical pulses (see [7] for more details).

A heuristic algorithm for generating practical test functions using MATLAB procedures was presented in [6]. First it has been shown that ideal test functions cannot be generated by differential equations, being emphasized the fact that differential equations can only generate functions similar to test functions (defined as practical test functions). Then a step-by-step algorithm for designing the most simple differential equation able to generate a practical test function was presented, based on the invariance properties of the differential equation and on standard MATLAB procedures. The result of the algorithm consists in a system working at the stability limit from initial null conditions, on limited time intervals, the external signal representing the free term in the differential equation corresponding to the input of the oscillating system. Such a system could be built using standard components and operational amplifiers. However, the previously mentioned study [6] did not investigate the behavior of such an oscillating system for an input represented by a short-step pulse. These aspects will be studied in this paper. Finally, supplementary resonance aspects (appearing when the input frequency is close to the working frequency of the oscillating second-order system) will be also presented. 


\section{Modeling transitions by practical test-functions integral aspects}

While this study is based on robust integral procedures of practical test-functions for certain time intervals, certain basic integral aspects of practical test functions should be mentioned. These aspects are useful for modeling smooth transitions from a certain function of time to another on a limited time interval [6].

From basic mathematics, it is known that the product $\varphi(t) g(t)$ between a function $g(t)$ which belongs to $C^{\infty}$ class and a test-function $\varphi(t)$ which differs to zero on $(a, b)$ is also a test-function because

(a) it differs to zero only on the time interval $(a, b)$ where $\varphi(t)$ differs to zero (if $\varphi(t)$ is null, then the product $\varphi(t) g(t)$ is also null);

(b) the function $\varphi(t) g(t)$ belongs to the $C^{\infty}$ class of functions, while a derivative of a certain order $k$ can be written as

$$
(\varphi(t) g(t))^{(k)}=\sum_{p=0}^{k} C_{k}^{p} \varphi(t)^{(p)} g(t)^{(k-p)}
$$

(a sum of terms represented by a product of two continuous functions).

Yet for practical cases (when phenomena must be represented by differential equations), the $\varphi(t)$ test functions must be replaced by a practical test functions $f(t) \in C^{n}$ on $R$ (for a finite $n$-considered from now on as representing the order of the practical test function) having the following properties:

(a) $f$ is nonzero on $(a),(b)$,

(b) $f$ satisfies the boundary conditions $f^{(k)}(a)=f^{(k)}(b)=0$ for $k=0,1, \ldots, n$, and

(c) $f$ restricted to $(a, b)$ is the solution of an initial value problem (i.e., an ordinary differential equation on $(a, b)$ with initial conditions given at some point in this interval).

The generation of such practical test functions is based on the study of differential equations satisfied by these test functions, with the initial moment of time chosen at a time moment close to the $t=a$ moment of time (when the function begins to present nonzero values).

By using these properties of practical test-functions, we obtain the following important result for a product $f(t) g(t)$ between a function $g(t)$ which belongs to $C^{\infty}$ class and a practical test-function of $n$ order $f(t)$ which differs to zero on $(a, b)$ :

\section{General property for product}

The product $g(t) f(t)$ between a function $g(t) \in C^{\infty}$ and a practical test-function $f$ of order $n$ is represented by a practical test function of order $n$.

This is a consequence of the following two properties:

(a) the product $g(t) f(t)$ differs to zero only on the time interval $(a, b)$ on which $f(t)$ differs to zero; 
(b) the derivative of order $k$ for the product $g(t) f(t)$ is represented by the sum

$$
(f(t) g(t))^{(k)}=\sum_{p=0}^{k} C_{k}^{p} f(t)^{(p)} g(t)^{(k-p)}
$$

which is a sum of terms representing products of two continuous functions for any $k \leq n$, $(n$ being the order of the practical test-function $f)$-only for $k>n$ discontinuous functions can appear in the previous sum.

Now we will begin to study the integral properties of practical test functions of certain order. For this, we note that the integral $\varphi(t)$ of a test function $\phi(t)$ (which differs to zero on $(a, b)$ interval) is a constant function on the time intervals $(-\infty, a]$ and $[b,+\infty)$; it presents a certain variation on the $(a, b)$ time interval, from a constant null value to a certain $\Delta$ quantity corresponding to the final constant value. Moreover, all derivatives of order $k \leq n+1$ for the integral function $F(t)$ are equal to zero for $t=a$ and $t=b$ (this can be easily checked by taking into account that all derivatives of order $p$ for $f(t)$ are equal to zero at these time moments, for $p \leq n$, and a derivative of order $p$ for $f(t)$ corresponds to a derivative of order $p+1$ for function $F(t)$, the integral function of $f(t))$. This suggests the possibility of using such integral functions for modeling smooth transitions from a certain state to another in different kind of applications, when almost all derivatives of a certain function are equal to zero at the initial moment of time.

For modeling such a transition, we analyze the general case when a function $f$ and a finite number of its derivatives $f^{(1)}, f^{(2)}, \ldots f^{(n)}$ present variations from null values to values $\Delta, \Delta_{1}, \Delta_{2}, \ldots \Delta_{n}$ on the time interval $[-1,1]$. We begin by looking for a function $f_{n}$ which should be added to the null initial function so as to obtain a variation $\Delta_{n}$ for the derivative of $n$ order.

By multiplying the bump-like function

$$
\varphi(\tau)= \begin{cases}C \exp \left(\frac{1}{\tau^{2}-1}\right), & \text { if } \tau \in(-1,1), \\ 0, & \text { otherwise }\end{cases}
$$

(a test-function on $[-1,1]$ ) with the variation $\Delta_{n}$ of the derivative of $n$ order and by integrating this product $n+1$ times we obtain

(i) after the first integration: a constant value equal to $\Delta_{n}$ at the time moment $t=1$ (while the integral of the bump-like test function on $[-1,1]$ is equal to 1 ), and a null variation on $(1,+\infty)$;

(ii) after the second integration (when we integrate the function obtained at previous step): a term equal to $\Delta_{n}(t-1)$ and a term equal to a constant value $c_{11}$ (a constant of integration) on the time interval $(1,+\infty)$;

(iii) after the $n+1$ integration: a term equal to $\Delta_{n}(t-1)^{n} / n$ ! and a sum of terms having the form $c_{1 i}(t-1)^{i} / i$ ! for $i \in N, i<n$ ( $c_{n i}$ being constants of integration) on the time interval $(1,+\infty)$.

All previous constants of integration are determined by integrating the test function on $[-1,1]$. The procedure continues by looking for the other functions $f_{n-1}, f_{n-2} \ldots$ which must be added to the initial null function. However, we must take care to the fact that the 
function $f_{n}$ previously obtained has nonzero variations $d_{n-1}, d_{n-2}, \ldots d_{1}$ for its derivatives of order $n-1, n-2, \ldots 1$ on the working interval and so we must subtract these values from the set $\Delta_{n-1}, \Delta_{n-2}, \ldots \Delta_{1}$ before passing to the next step.

Then we multiply the bump-like function with the corrected value

$$
\Delta_{n-1}^{\prime}=\Delta_{n-1}-d_{n-1}
$$

and by integrating this product $n$ times we obtain in a similar manner a function with a term equal to $\Delta_{n-1}^{\prime}(t-1)^{n-1} /(n-1)$ ! and a sum of terms having the form $c_{2 i}(t-1)^{i} / i$ ! for $i \in N, i<n-1$ ( $c_{n i}$ being constants of integration) on the time interval $(1,+\infty)$. It can be noticed that the result obtained after $n$ integration possess the $n-1$ order derivative equal to $\Delta_{n-1}^{\prime}$, a smooth transition for this derivative from the initial null value being performed. So the second function which must be added to the initial null function is the integral of $n-1$ order for the bump-like function multiplied by this variation $\Delta_{n-1}-d_{n-1}$ (the function being noted as $f_{n-1}$ ). This function $f_{n-1}$ has a null value for the derivative of $n$ order for $t>1$, so the result obtained at first step is not affected. We must take care again to the fact that the function $f_{n-1}$ previously obtained has nonzero variations $d_{n-1}^{1}, d_{n-2}^{1}, \ldots d_{1}^{1}$ for its derivatives of order $n-$ $1, n-2, \ldots 1$ and so we must once again subtract these values from the previously corrected set $\Delta_{n-1}-d_{n-1}, \Delta_{n-2}-d_{n-2}, \ldots \Delta_{1}-d_{1}$ before passing to the next step. Finally we obtain all functions $f_{n+1}, f_{n}, \ldots f_{1}$ which represent the terms of function $f$ modeling the smooth transition from an initial null function to a function having a certain set of variations for a finite number of its derivatives on a small time interval. The procedure can be also applied for functions possessing a finite number of derivatives within a certain time interval by time reversal $(t$ being replaced with $-t$ ). More details regarding possible applications of such a procedure can be found in [6].

\section{The oscillating second-order system for the case of short-step inputs}

After presenting basic aspects regarding integral properties of practical test-functions, we will analyze the behavior of a system able to generate a practical test-function for signal processing when its command is represented by a short-step pulse. Unlike aspects presented in previous paragraph, the step change appears for the command function $u(t)$, and the dynamical behavior on a limited time interval should be performed. We are searching for a robust integral procedure (with null values of the function which is integrated at the beginning and at the end of the interval of integration) so as the sampled values to be further processed for determining the amplitude and the time length of the short-step pulse.

For a robust filtering and sampling procedure based on an integration on a limited time interval, a search for a system having the following property was performed in a rigorous manner in [8]: starting to work from initial null conditions, for a unity step input it must generate an output and a derivative of this output equal to zero at a certain moment of time (the condition for the derivative of the output to be equal to zero has been added so as the slope and the first derivative of the slope for the signal which is integrated to be equal to zero at the sampling moment of time, when the integration is interrupted). It was finally shown that the simplest structure possessing such properties is represented by an oscillating second-order system having the transfer function

$$
H_{\mathrm{osc}}=\frac{1}{T_{0}^{2} s^{2}+1}
$$


receiving a step input and working on the time interval $\left[0,2 \pi T_{0}\right]$. For initial conditions equal to zero, the response of the oscillating system at a step input with amplitude $A$ will have the form

$$
y(t)=A\left(1-\cos \left(\frac{t}{T_{0}}\right)\right)
$$

By integrating this result on the time interval $\left[0,2 \pi T_{0}\right]$, we obtain the result $2 \pi A T_{0}$, and we can also notice that the quantity which is integrated and its slope are equal to zero at the end of the integration period. Thus the influence of the random variations of the integration period (generated by the switching phenomena) is practically rejected.

This oscillating system attenuates about $\left(\omega / \omega_{0}\right)^{2}$ times such an input, and the influence of the integrator consists in a supplementary attenuation of about

$$
\left[(1 /(2 \pi))\left(\omega / \omega_{0}\right)\right]
$$

times. The oscillations having the form

$$
y_{\mathrm{osc}}=a \sin \left(\omega_{0} t\right)+b \cos \left(\omega_{0} t\right)
$$

generated by the input alternating component have a lower amplitude and give a null result after an integration over the time interval $\left[0,2 \pi T_{0}\right]$.

These results have shown that such a structure provides practically the same performances as a structure consisting of an asymptotically stable second-order system and an integrator (response time of about $6 T_{0}$, an attenuation of about $(1 / 6)\left(\omega / \omega_{0}\right)^{3}$ times for an alternating component having frequency $\omega$ ), moreover being less sensitive at the random variations of the integration period. For restoring the initial null conditions after the sampling procedure (at the end of the working period), some electronic devices must be added. Yet the previous analysis is valid for extended step inputs, which are active on the whole working interval (the integration period).

We will continue the analysis of this structure by considering that the input is represented by a unity short-step pulse (instead of a unity step-pulse) which differs to zero on the time interval $[0, \tau]$. This means that the input $u$ can be represented under the following form:

$$
\begin{array}{ll}
u(t)=1, & \text { for } t \in[0, \tau], \\
u(t)=0, & \text { for } t>\tau,
\end{array}
$$

or, using the Heaviside function

$$
u(t)=h(\tau-t) \text { for } t \in[0, \infty),
$$

where $h(\tau)$ corresponds to the function $1 / s$ if we apply the Laplace transformation.

The transfer function of the second-order oscillating system is

$$
H(s)=\frac{1}{T_{0}^{2} s^{2}+1}
$$


On the time interval $[0, \tau]$, the output of the second-order oscillating system is represented (using the Laplace transformation) as

$$
y(s)=H(s) u(s)=\frac{1}{T_{0}^{2} s^{2}+1} \frac{1}{s}
$$

which corresponds to the output

$$
y(t)=\left(1-\cos \left(\frac{t}{T_{0}}\right)\right)
$$

which can be written as

$$
y(t)=1-\cos \left(\omega_{0} t\right)
$$

where $\omega_{0}=2 \pi / T_{0}$. When the action of the external unity pulse ceases (for $t=\tau$ ) the output $y(t)$ is

$$
y(\tau)=1-\cos \left(\omega_{0} \tau\right)
$$

and the derivative of $y(t)$ is

$$
y^{\prime}(\tau)=\omega_{0} \sin \left(\omega_{0} \tau\right)
$$

These values, $y(\tau)$ and $y^{\prime}(\tau)$, represent the initial values for the free oscillations of the second-order oscillating system generated for $t>\tau$ (when the input command $u(t)=0$ ). These free oscillations have the angular velocity $\omega_{0}$, and thus the output $y(t)$ for $t>\tau$ will have the form

$$
y(t)=C \sin \left(\omega_{0} t+\phi\right), \quad \text { for } t>\tau
$$

The quantities $C$ and $\phi$ (amplitude and initial phase of free oscillations) should be determined using the initial conditions for $t=\tau$ :

$$
y(\tau)=1-\cos \left(\omega_{0} \tau\right), \quad y^{\prime}(\tau)=\omega_{0} \sin \left(\omega_{0} \tau\right)
$$

This implies

$$
1-\cos \left(\omega_{0} \tau\right)=C \sin \left(\omega_{0} \tau+\phi\right), \quad \omega_{0} \sin \left(\omega_{0} \tau\right)=C \omega_{0} \cos \left(\omega_{0} \tau+\phi\right) .
$$

By dividing the second equality with $\omega_{0}$, squaring both equalities and summing lefthand sides and right-hand sides of both squared equalities we obtain

$$
\left[1-\cos \left(\omega_{0} \tau\right)\right]^{2}+\sin ^{2}\left(\omega_{0} \tau\right)=C^{2}
$$

and $\left(\right.$ while $\left.\sin ^{2}\left(\omega_{0} \tau\right)+\cos ^{2}\left(\omega_{0} \tau\right)=1\right)$

$$
2-2 \cos \left(\omega_{0} \tau\right)=C^{2} .
$$


While

$$
\cos \left(\omega_{0} \tau\right)=1-2 \sin ^{2}\left(\frac{\omega_{0} \tau}{2}\right)
$$

by substituting $\cos \left(\omega_{0} \tau\right)$ with the right-hand side of the above equality, the result is

$$
4 \sin ^{2}\left(\frac{\omega_{0} \tau}{2}\right)=C^{2}
$$

It results

$$
C=2 \sin \left(\frac{\omega_{0} \tau}{2}\right)
$$

( $C$ is a positive quantity, because $\omega_{0} \tau \in[0,2 \pi] \rightarrow \omega_{0} \tau / 2 \in[0, \pi] \rightarrow \sin \left(\omega_{0} \tau / 2\right) \geq 0$ ). The phase $\phi$ can be obtained using the two equations determined by the initial conditions:

$$
1-\cos \left(\omega_{0} \tau\right)=C \sin \left(\omega_{0} \tau+\phi\right), \quad \sin \left(\omega_{0} \tau\right)=C \cos \left(\omega_{0} \tau+\phi\right)
$$

(the second equation resulting by dividing previous equation of $y^{\prime}(\tau)$ to $\left.\omega_{0}\right)$.

By dividing left-hand side of first equality to left-hand side of second equality, and right-hand side of first equality to right-hand side of second equality, it results

$$
\frac{1-\cos \left(\omega_{0} \tau\right)}{\sin \left(\omega_{0} \tau\right)}=\tan \left(\omega_{0} \tau+\phi\right)
$$

Using equalities

$$
\cos \left(\omega_{0} \tau\right)=1-2 \sin ^{2}\left(\frac{\omega_{0} \tau}{2}\right), \quad \sin \left(\omega_{0} \tau\right)=2 \sin \left(\frac{\omega_{0} \tau}{2}\right) \cos \left(\frac{\omega_{0} \tau}{2}\right)
$$

it results by substituting $\cos \left(\omega_{0} \tau\right), \cos \left(\omega_{0} \tau\right)$ with the above expressions

$$
\tan \left(\frac{\omega_{0} \tau}{2}\right)=\tan \left(\omega_{0} \tau+\phi\right)
$$

So $\phi$ is obtained as

$$
\phi=-\left(\frac{\omega_{0} \tau}{2}\right)
$$

and the output $y(t)$ corresponding to the free oscillations of the system for $t>\tau$ (when the action of the external short-step command $u$ has ceased) can be written as

$$
y(t)=2 \sin \left(\frac{\omega_{0} \tau}{2}\right) \sin \left(\omega_{0} t-\frac{\omega_{0} \tau}{2}\right)
$$




\section{Algorithm for detecting short-step pulses}

While the signal processing system is linear, in case of a short-step pulse of amplitude $A$ defined on time interval $[0, \tau]$ the output $y(t)$ of the system will be multiplied by $A$ as related to the output obtained in case of a unity short-step input (presented in previous paragraph). Thus, the output $y(t)$ will be

$$
\begin{gathered}
A\left(1-\cos \left(\omega_{0} t\right)\right), \quad \text { for } t \in[0 . \tau], \\
y(t)=2 A \sin \left(\frac{\omega_{0} \tau}{2}\right) \sin \left(\omega_{0} t-\frac{\omega_{0} \tau}{2}\right), \quad \text { for } t>\tau .
\end{gathered}
$$

This output, $y(t)$, is equal to zero at two time moments $t 1$ and $t 2$ after time moment $\tau$. At time moment $t 1$,

$$
\left(\omega_{0} t 1-\frac{\omega_{0} \tau}{2}\right)=\pi
$$

and at time moment $t 2$,

$$
\left(\omega_{0} t 2-\frac{\omega_{0} \tau}{2}\right)=2 \pi
$$

These imply that

$$
\begin{aligned}
& t 1=\frac{\tau}{2}+\frac{\pi}{\omega_{0}}, \\
& t 2=\frac{\tau}{2}+2\left(\frac{\pi}{\omega_{0}}\right) .
\end{aligned}
$$

We must check whether both $t 1, t 2$ are greater than $\tau$. First we check the inequality $t 1=\tau / 2+\pi / \omega_{0}>\tau$. This is equivalent to $\tau / 2<\pi / \omega_{0}, \tau<2 \pi / \omega_{0}=T_{0}$, where $T_{0}$ represents the period of the second-order oscillating system. It is obvious that $\tau<T_{0}$, while we have considered that the short-step pulse has nonzero values for $t \in\left(0, T_{0}\right)$. Thus $t 1>\tau$, and while $t 2>t 1$ it results that $t 2>t 1>\tau$.

The signal processing system will perform the integration of output $y(t)$ on two different time intervals. The first value $I 1$ is obtained by an integration of $y(t)$ on the time interval $[0, t 2]$. It results

$$
\begin{aligned}
& I 1=\int_{0}^{t 2} y(t) d t=\int_{0}^{\tau} A\left(1-\cos \left(\omega_{0} t\right)\right) d t+\int_{\tau}^{t 2} 2 A \sin \left(\frac{\omega_{0} \tau}{2}\right) \sin \left(\omega_{0} t-\frac{\omega_{0} \tau}{2}\right) d t, \\
& I 1=A\left(\tau-\frac{1}{\omega_{0}} \sin \left(\omega_{0} \tau\right)+2 A \frac{1}{\omega_{0}} \sin \left(\frac{\omega_{0} \tau}{2}\right)\left(-\cos (2 \pi)+\cos \left(\frac{\omega_{0} \tau}{2}\right)\right),\right.
\end{aligned}
$$

$\left(\right.$ while $\left.\omega_{0} t 2=2 \pi\right)$. Then we obtain

$$
I 1=A \tau-A \frac{1}{\omega_{0}} \sin \left(\omega_{0} \tau\right)+A \frac{2}{\omega_{0}} \sin \left(\frac{\omega_{0} \tau}{2}\right)\left(\cos \left(\frac{\omega_{0} \tau}{2}\right)-1\right)
$$


which can be finally written as

$$
I 1=A \tau-A \frac{2}{\omega_{0}} \sin \left(\frac{\omega_{0} \tau}{2}\right) .
$$

The second integral $I 2$ is performed by integrating $y(t)$ on the time interval $[t 1, t 2]$. On this time interval, the output $y(t)$ is represented by a free oscillation

$$
y(t)=2 A \sin \left(\frac{\omega_{0} \tau}{2}\right) \sin \left(\omega_{0} t-\frac{\omega_{0} \tau}{2}\right)
$$

so $I 2$ is determined by

$$
I 2=\int_{t 1}^{t 2} 2 A \sin \left(\frac{\omega_{0} \tau}{2}\right) \sin \left(\omega_{0} t-\frac{\omega_{0} \tau}{2}\right) d t
$$

and taking into account that

$$
\begin{aligned}
& \omega_{0} t-\frac{\omega_{0} \tau}{2}=\pi, \quad \text { for } t=t 1, \\
& \omega_{0} t-\frac{\omega_{0} \tau}{2}=2 \pi, \quad \text { for } t=t 2,
\end{aligned}
$$

it results $I 2$ as

$$
I 2=-\frac{4 A}{\omega_{0}} \sin \left(\frac{\omega_{0} \tau}{2}\right)
$$

The values $I 1, I 2$ allow us to determine $A, \tau$ by robust integrations (the values of the function $y(t)$ which is integrated are zero at the beginning and the end of the time interval used for integration). A quantity $I 0$ can be determined as

$$
I 0=I 1-\frac{I 2}{2}=A \tau
$$

and a quantity $R$ can be determined as

$$
R=-\frac{I 2}{2 I 0}=\frac{\left(4 A / \omega_{0}\right) \sin \left(\omega_{0} \tau / 2\right)}{2 A \tau}
$$

which can be written as

$$
R=\frac{\sin \left(\omega_{0} \tau / 2\right)}{\omega_{0} \tau / 2}=\operatorname{sinc}\left(\frac{\omega_{0} \tau}{2}\right) .
$$

This means that after performing the robust integration of $y(t)$ in order to obtain the sampled values $I 1, I 2$ we can compute $I 0=I 1-I 2 / 2$ and then $I 0=-I 2 /(2 I 0)$. While

$$
I 0=\operatorname{sinc}\left(\frac{\omega_{0} \tau}{2}\right)
$$

we can determine the time interval $\tau$ of the received short-step pulse using $I 0$ and a mathematical memory (the quantity $\omega_{0}$ being known).

Next quantity $A$ (the amplitude of this received short-step pulse) can be determined using $\tau$ (determined at previous computation) and $I 0=A \tau$. 


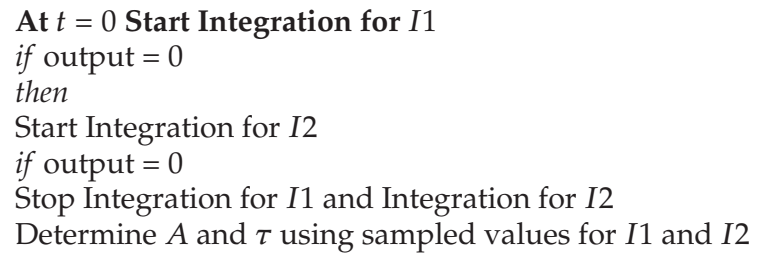

Algorithm 1

Taking into account the previous considerations, the algorithm for detecting short-step pulses consists in the steps shown in Algorithm 1.

\section{The problem of initial conditions and resonance aspects}

In previous paragraphs has been analyzed the case when a certain short-step pulse is received at the beginning of a working interval-this pulse presenting nonzero values on a limited time interval $(0, \tau)$. Yet in applications, the signal processing system can receive a sequence of step pulses with different time lengths. Due to this reason, the whole procedure must be adapted for the case when a certain step input with amplitude $D$ is received on a certain time interval $(0, \sigma)$ (the first part of the working interval) and a different step input with amplitude $B$ appears on the time interval $\left[\sigma, T_{0}\right]$ (the second part of the time interval).

Under these circumstances the output $y(t)$ could be represented by

$$
\begin{aligned}
& y(t)=D\left(1-\cos \left(\omega_{0} t\right)\right), \quad \text { for } t<\sigma, \\
& y(t)=B\left(1-\cos \left(\omega_{0}(t-\sigma)\right)\right)+y_{\text {osc }}, \text { for } t \geq \sigma,
\end{aligned}
$$

where

$$
y_{\mathrm{osc}}=2 D \sin \left(\frac{\omega_{0} \sigma}{2}\right) \sin \left(\omega_{0} t-\frac{\omega_{0} \sigma}{2}\right)
$$

represents the free oscillations of the system generated by the short-step pulse with amplitude $D$ (which has ceased its action at time moment $\sigma$ ). It can be noticed that it is very difficult to analyze these outputs by performing certain integral operations in order to determine the parameters $D, B, \sigma$. However, the whole procedure can be simplified in a significant manner if we observe that the amplitude $D$ of the first step input can be predicted by the signal processing system (by considering that the pulse with amplitude $D$ has been received by the system on a previous working interval and its action continues at the beginning of the analyzed working interval). This suggests the possibility of adjusting the input command for the second-order oscillating system by subtracting quantity $D$ from $u(t)$ and thus the output of the oscillating system will become

$$
\begin{aligned}
& y(t)=0, \quad \text { for } t<\sigma, \\
& y(t)=(B-D)\left(1-\cos \left(\omega_{0}(t-\sigma)\right)\right), \quad \text { for } t \geq \sigma .
\end{aligned}
$$

The quantity $y_{\text {osc }}$ vanishes while the oscillating second-order system has initial null conditions and a null command for $t<\sigma$. 
At first sight it looks like the system receives a nonzero command at the end of the working time interval and the results presented in previous paragraph cannot be applied. Yet we can observe that an integration performed on the first time interval $[0, \sigma]$ generates a null result. Due to this reason, we have to adjust the integration procedure by

(i) starting the integration for $I 1$ and $I 2$ at the beginning of a new working cycle, with the input command considered as $w(t)=u(t)-A$ (the previous value for the amplitude of the received pulse is subtracted from received pulse $u(t)$ );

(ii) if a step variation of amplitude for the input command $w(t)$ is detected then

(a) stop the action of adjusted input command $w(t)=u(t)-A$ upon the oscillating system at time moment $T_{0}$ (the output $y(t)$ of the system will be represented by free oscillations),

(b) continue the integration for $I 1$ and $I 2$ after the time moment $T_{0}$ (which would have been the end of the working cycle in case that the step variation of input has been not detected);

(iii) stop the integration procedure for $I 1$ when $y(t)$ first time equals zero and the integration procedure for $I 2$ when $y(t)$ second time equals zero.

Note that the time moments when the integration procedures cease are not affected by noise, while the output $y(t)$ is represented just by free oscillations of the secondorder system (after the time moment $T_{0}$ the action of $u(t)$ upon the system ceases);

(iv) Determine $\sigma$ using

$$
\begin{aligned}
& I 0=-I 2 /(2 I 0), \quad I 0=\operatorname{sinc}\left(\frac{\omega_{0} \tau}{2}\right), \quad T_{0}-\sigma=\tau, \\
& I 0=A \tau, \quad B-D=A, \quad B=D+A .
\end{aligned}
$$

Note: by translating the time origin from the beginning of a working interval to the time moment when the step change $(B-D)$ appears, we can consider that a short-step pulse with amplitude $(B-D)$ acts upon the second-order system at time moment zero, from null initial conditions, on the time interval $\left(0, T_{0}-\sigma\right)$ - thus the quantities $\tau$ and $A$ corresponding, respectively, to the time length of the received short-step pulse and to its amplitude in previous paragraph should be replaced by $T_{0}-\sigma$ and $(B-D)$.

All presented aspects are valid if the system receives a step pulse (which can be represented by an extended step pulse as presented in [1]) or by a short-step pulse (as presented in this study, in previous paragraphs). Filtering properties of the second-order oscillating system were studied in [1]. However, we must study also resonance aspects. The second-order system being an oscillating system, resonance aspects (appearing when the input $u(t)$ is represented by an alternating function $A \sin (\omega t+\varphi)$ with $\left.\omega \approx \omega_{0}\right)$ are very important. Instead of damped proper oscillations of angular frequency $\omega_{0}$ with zero limit (as for an asymptotic stable second-order system), some proper oscillations of angular frequency $\omega_{0}$ and with constant amplitude can be noticed as term in $y(t)$. These are added to the oscillations with angular frequency $\omega$ generated by the command function $u(t)$, both having a higher amplitude inversely proportional to $\omega^{2}-\omega_{0}^{2}$. If $\omega=\omega_{0}$ (the limit case), for a command function

$$
u(t)=A \sin \left(\omega_{0} t+\varphi_{0}\right)
$$


the output $y(t)$ is represented by

$$
y(t)=E_{1} t \sin \left(\omega_{0} t+\varphi_{1}\right)+E_{2} \sin \left(\omega_{0} t+\varphi_{2}\right) .
$$

This function is hard to be processed by a signal processing system, working on a limited time interval. Moreover, in applications it is possible for the input $u(t)$ to be represented by a sum of alternating functions with different angular frequencies $\omega_{i} \approx \omega_{0}$. However, a signal processing procedure can be established for the case when $u(t)$ is represented by a sum of alternating functions with angular frequencies $\omega_{i} \approx \omega_{0}$ by taking into account the fact that for an input $u(t)$ represented by an alternating function $A \sin (\omega t+\varphi)$ the amplitude $E$ of oscillations with angular frequency $\omega$ generated by this command function $u(t)$ is determined by

$$
E(\omega)=\frac{A \omega_{0}^{2}}{\omega^{2}-\omega_{0}^{2}}
$$

which is a very sharp function. For this reason we can consider that in the case when the input $u(t)$ is represented by a sum of oscillations with different angular frequencies $\omega_{i} \approx \omega_{0}$, the output $y(t)$ of the second-order system will be represented by an oscillation with the angular frequency $\omega_{j}$ closest to $\omega_{0}$ (generated by the received oscillation with angular frequency $\omega_{j}$ ) and a proper oscillation of the second-order system (with angular frequency $\omega_{0}$ ). Thus $y(t)$ could be represented by a sum of two oscillations.

The result of an integration of this output $y(t)$ on the working interval $\left[0,2 \pi T_{0}\right]$ would depend just on the oscillation with angular frequency $\omega_{j}$, while the oscillation with angular frequency $\omega_{0}$ (with time period $T_{0}$ ) gives a null result by an integration on this period. The result of this integration could be used for determining certain parameters for the received oscillation with angular frequency $\omega_{j}$. However, such an integration is not a robust integration, while the signal which is integrated is not equal to zero at the end of this working interval. A possible solution of this problem would consist in disconnecting the input signal after a certain time interval, so as to analyze (using robust integrations) the free oscillations of the second-order system after this moment (as presented in previous paragraph). A faster procedure could consist in a previous adjustment of initial conditions, so as the free oscillations not to appear.

Theoretically, this can be done by using a set of identical oscillating second-order systems (receiving the same input command $u(t)$ ) with initial conditions adjusted to different values. The system generating a single oscillation with angular frequency $\omega_{j}$ would be selected by checking the following condition:

$$
\frac{d^{2} y}{d t^{2}}+\omega_{j}^{2} y=0
$$

However, the adjustment of two initial conditions at different values for a second-order system requires a large number of identical oscillators. Due to this reason, this method is inconvenient. A better choice would be represented by a delay systems of first order, with transfer function

$$
H(s)=\frac{s}{s \sin \varphi+\omega_{j} \cos \varphi}
$$


would transform a received oscillation $\sin \left(\omega_{j} t+\varphi\right)$ according to

$$
V(s)=H(s) U(s)=\frac{s}{s \sin \varphi+\omega_{j} \cos \varphi} \frac{s \sin \varphi+\omega_{j} \cos \varphi}{s^{2}+\omega_{j}^{2}}=\frac{s}{s^{2}+\omega_{j}^{2}}
$$

which corresponds to an output $v(t)=\cos \left(\omega_{0} t\right)$. If this function $v(t)$ represents the command for the second-order oscillating system, the output $y(t)$ (for initial null conditions) will be

$$
y(t)=(1 / 2) \omega_{j} t \sin \left(\omega_{j} t\right)
$$

when $\omega_{j}$ is very close to $\omega_{0}$ and can be approximated by this quantity. The function $y(t)$ presented in previous equation is suitable for a sequence of robust integration procedures on half-period time intervals:

$$
\left[0, \pi / \omega_{j}\right],\left[\pi / \omega_{j}, 2 \pi / \omega_{j}\right], \text { etc. }
$$

(it presents null values at the beginning and at the end of these intervals integration). The results of these procedures are proportional to the amplitude $A$ of received oscillation with angular frequency $\omega_{j}$ (as can be easily noticed).

Unlike the possible solution based on adjustment of two initial conditions, this procedure requires a set of signal processing systems composed of different time-delaying systems adjusted according to a single parameter (the phase $\phi$, while $\omega_{j}$ is supposed to be known) and identical second-order oscillating systems starting to work from initial null conditions. The number of required systems is less than in previous case, while a single parameter has to be adjusted at different values (the quantity $\varphi$ in the time-delay systems). The corresponding output is selected by checking whether the results of these procedures (considered as positive quantities) vary according to a linear mathematical law (as required by the integration of $y(t)=(1 / 2) \omega_{j} t \sin \left(\omega_{j} t\right)$ on a sequence of half-period time intervals).

One major disadvantage of this method has to be mentioned: the function $y(t)=$ $(1 / 2) \omega_{j} t \sin \left(\omega_{j} t\right)$ equals zero at the beginning of signal processing time interval $(t=0)$ and presents a small slope. So an extended time interval is necessary for obtaining significant results using robust integration procedures. If we need a fast signal processing, we can simply use a set of identical oscillating second-order systems, with different initial conditions. The greatest amplitude for the output oscillation (considered as a sum of an oscillation with angular frequency $\omega_{j}$ and an oscillation with angular frequency $\omega_{0}, \omega_{j} \approx \omega_{0}$ ) corresponds to the case when the two oscillations are in-phase. By detecting the output presenting inphase oscillations, we can establish the amplitude and phase for the received signal using the initial conditions for the second-order system generating this output.

\section{Conclusions}

An important aspect in modeling dynamic phenomena consists in measuring with higher accuracy some physical quantities corresponding to the dynamic system. Yet for measurements performed on limited time interval at high working frequency, certain intelligent methods should be added. The high working frequency requires that the measurement and data processing time interval should be greater than the time interval when the step input is received, so as to allow an accurate measurement. This paper has shown that an intelligent processing method based on oscillating second-order systems working on limited 
time interval can differentiate between large-step inputs (which are active on the whole limited time interval) and short-step inputs (which are active on a time interval shorter than the limited working period). Some resonance aspects (appearing when the input frequency is close to the working frequency of the oscillating second-order system) were also presented. Possible applications could be represented by processing the electric signal generated by transducers [9] and by advanced modeling of traffic network [10].

\section{Acknowledgments}

This study was performed by first author (from FILS Department, Politehnica University, Bucharest) during an internship at Corner Soft Technologies, Bucharest (research coordinated by the second author).

\section{References}

[1] C. Toma, "An extension of the notion of observability at filtering and sampling devices," in Proceedings of the International Symposium on Signals, Circuits and Systems (ISSCS '05), p. 233, Iasi, Romania, July 2005.

[2] Y. Sun and J. K. Fidler, "Synthesis and performance analysis of universal minimum component integrator-based IFLF OTA-grounded capacitor filter," IEE Proceedings: Circuits, Devices and Systems, vol. 143, no. 2, pp. 107-114, 1996.

[3] S. L. Smith and E. Sánchez Sinencio, "Low voltage integrators for high frequency cmos filters using current mode techniques," IEEE Transactions on Circuits and Systems II, vol. 43, no. 1, pp. 39-48, 1996.

[4] C. Cattani and J. Rushchitsky, Wavelet and Wave Analysis as applied to Materials with Micro or Nanostructure, vol. 74 of Series on Advances in Mathematics for Applied Sciences, World Scientific, Singapore, 2007.

[5] C. Cattani, "Connection coefficients of Shannon wavelets," Mathematical Modelling and Analysis, vol. 11, no. 2, pp. 117-132, 2006.

[6] G. Toma, "Practical test-functions generated by computer algorithms," in Proceedings of the International Conference on Computational Science and Its Applications (ICCSA '05), vol. 3482 of Lecture Notes in Computer Science, pp. 576-584, Singapore, May 2005.

[7] S. Pusca, "Invariance properties of practical test-functions used for generating asymmetrical pulses," in Proceedings of the International Conference on Computational Science and Its Applications (ICCSA '06), vol. 3980 of Lecture Notes in Computer Science, pp. 763-770, Glasgow, UK, May 2006.

[8] A. Toma, S. Pusca, and C. Morarescu, "Spatial aspects of interaction between high-energy pulses and waves considered as suddenly emerging phenomena," in Proceedings of the International Conference on Computational Science and Its Applications (ICCSA '06), vol. 3980 of Lecture Notes in Computer Science, pp. 839-846, Glasgow, UK, May 2006.

[9] E. Smeu, "Fast photodetectors speed-related problems," Romanian Journal of Optoelectronics, vol. 11, no. 1, p. $63,2003$.

[10] M. Li and S. C. Lim, "Modeling network traffic using generalized Cauchy process," Physica A, vol. 387, no. 11, pp. 2584-2594, 2008. 


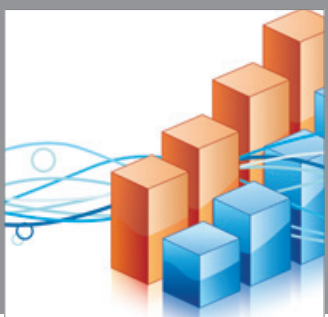

Advances in

Operations Research

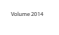

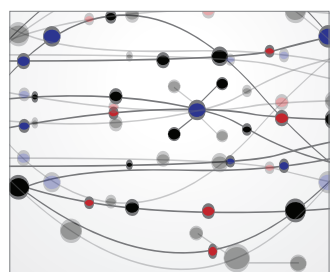

\section{The Scientific} World Journal
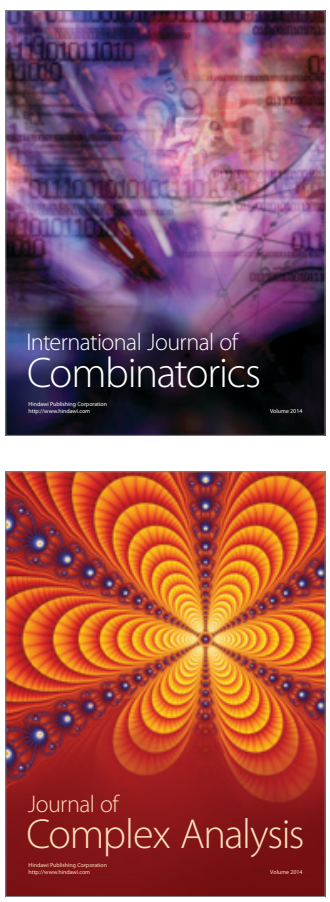

International Journal of

Mathematics and

Mathematical

Sciences
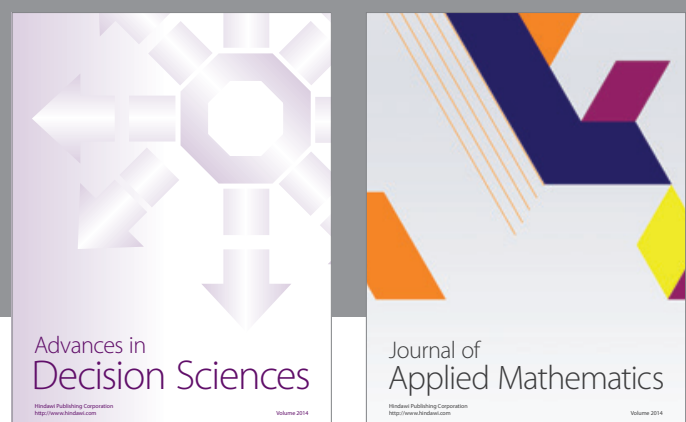

Journal of

Applied Mathematics
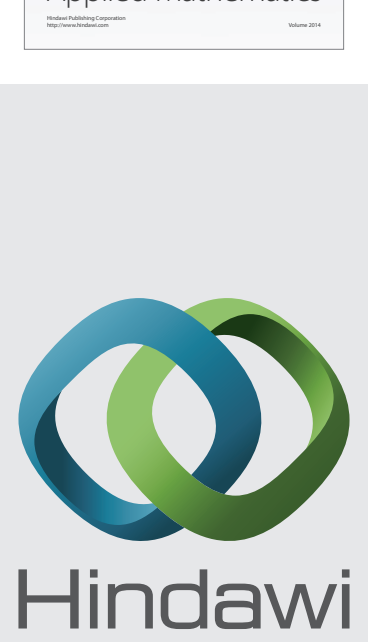

Submit your manuscripts at http://www.hindawi.com
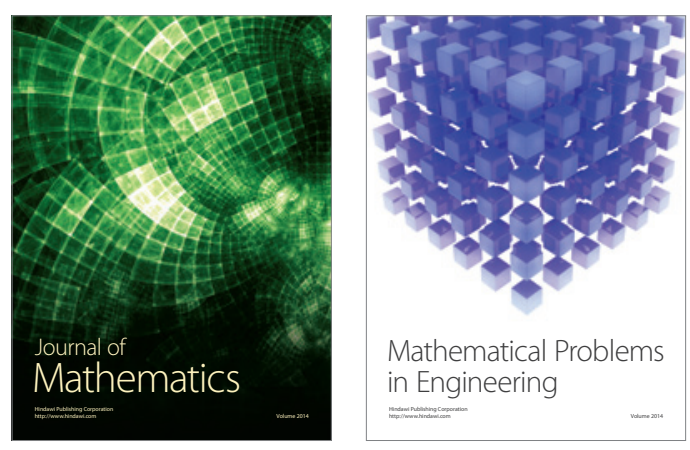

Mathematical Problems in Engineering
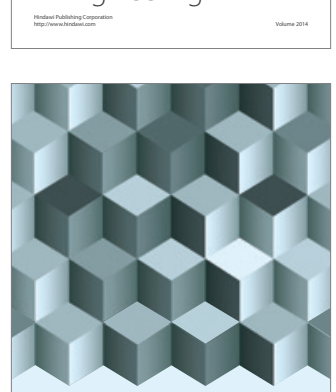

Journal of

Function Spaces
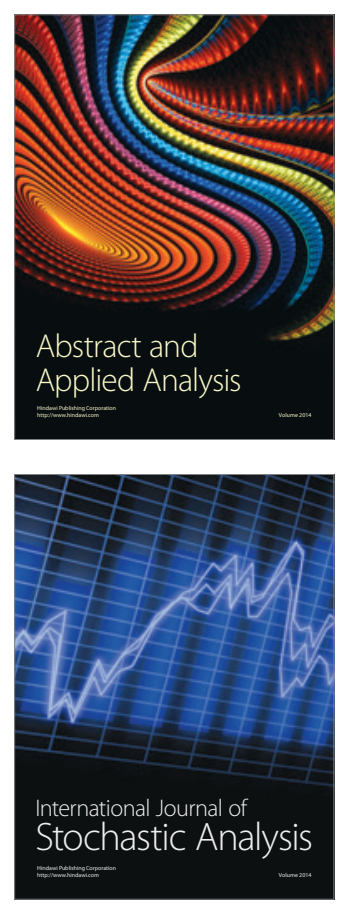

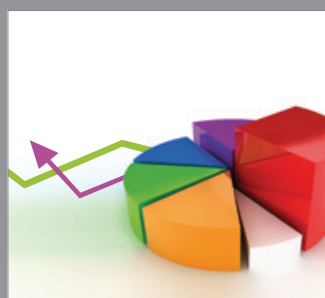

ournal of

Probability and Statistics

Promensencen
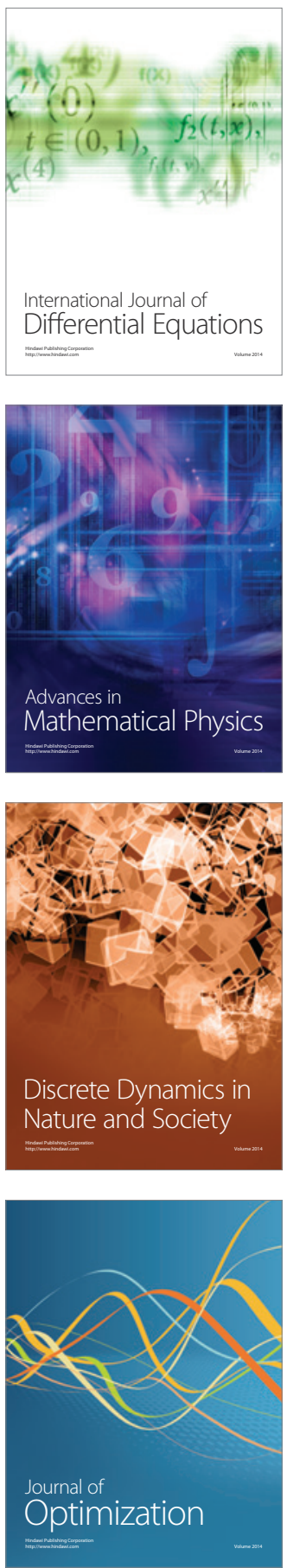